Article

\title{
Prognostic Impacts of D816V KIT Mutation and Peri-Transplant RUNX1-RUNX1T1 MRD Monitoring on Acute Myeloid Leukemia with RUNX1-RUNX1T1
}

\author{
Byung-Sik Cho ${ }^{1,2}$, Gi-June Min ${ }^{1,2}{ }^{1}$, Sung-Soo Park ${ }^{1,2} \mathbb{D}$, Silvia Park ${ }^{1,2}$, Young-Woo Jeon ${ }^{3}$, Seung-Hwan Shin ${ }^{4}$, \\ Seung-Ah Yahng ${ }^{5}$ (D) Jae-Ho Yoon ${ }^{1,2}$, Sung-Eun Lee ${ }^{1,2}$, Ki-Seong Eom ${ }^{1,2}$, Yoo-Jin Kim ${ }^{1,2}$, Seok Lee ${ }^{1,2}$, \\ Chang-Ki Min ${ }^{1,2}$, Seok-Goo Cho ${ }^{1}$, Dong-Wook Kim ${ }^{1,2}$, Jong-Wook Lee ${ }^{1}$, Myung-Shin Kim ${ }^{6} \mathbb{D}^{\text {, Yong-Goo Kim }}{ }^{6}$ \\ and Hee-Je Kim $1,2, *$ (D)
}

1 Department of Hematology, Catholic Hematology Hospital, Seoul St. Mary's Hospital, College of Medicine, The Catholic University of Korea, Seoul 06591, Korea; cbscho@catholic.ac.kr (B.-S.C.); beichest@catholic.ac.kr (G.-J.M.); sspark@catholic.ac.kr (S.-S.P.); silvia.park@catholic.ac.kr (S.P.); royoon@catholic.ac.kr (J.-H.Y.); lee86@catholic.ac.kr (S.-E.L.); dreom@catholic.ac.kr (K.-S.E.); yoojink@catholic.ac.kr (Y.-J.K.); leeseok@catholic.ac.kr (S.L.); ckmin@catholic.ac.kr (C.-K.M.); chosg@catholic.ac.kr (S.-G.C.); dwkim@catholic.ac.kr (D.-W.K.); jwlee@catholic.ac.kr (J.-W.L.)

2 Leukemia Research Institute, College of Medicine, The Catholic University of Korea, Seoul 06591, Korea

3 Department of Hematology, Yeouido St. Mary's Hospital, College of Medicine, The Catholic University of Korea, Seoul 06591, Korea; native47@catholic.ac.kr

4 Department of Hematology, Eunpyeong St. Mary's Hospital, College of Medicine, The Catholic University of Korea, Seoul 06591, Korea; chironhmt@catholic.ac.kr

check for updates

Citation: Cho, B.-S.; Min, G.-J.; Park, S.-S.; Park, S.; Jeon, Y.-W.; Shin, S.-H.; Yahng, S.-A.; Yoon, J.-H.; Lee, S.-E.; Eom, K.-S.; et al. Prognostic Impacts of D816V KIT Mutation and Peri-Transplant RUNX1-RUNX1T1 MRD Monitoring on Acute Myeloid Leukemia with RUNX1-RUNX1T1.

\section{Cancers 2021, 13, 336.}

https://doi.org/

10.3390/cancers 13020336

Received: 24 November 2020

Accepted: 14 January 2021

Published: 18 January 2021

Publisher's Note: MDPI stays neutral with regard to jurisdictional claims in published maps and institutional affiliations.

Copyright: (c) 2021 by the authors. Licensee MDPI, Basel, Switzerland. This article is an open access article distributed under the terms and conditions of the Creative Commons Attribution (CC BY) license (https:// creativecommons.org/licenses/by/ $4.0 /)$.
5 Department of Hematology, Incheon St. Mary's Hospital, College of Medicine, The Catholic University of Korea, Seoul 06591, Korea; saymd@catholic.ac.kr

6 Department of Laboratory Medicine, Seoul St. Mary's Hospital, College of Medicine, The Catholic University of Korea, Seoul 06591, Korea; microkim@catholic.ac.kr (M.-S.K.); yonggoo@catholic.ac.kr (Y.-G.K.)

* Correspondence: cumckim@catholic.ac.kr; Tel.: +82-2-2258-6054; Fax: +82-2-599-3589

Simple Summary: Acute myeloid leukemia (AML) with RUNX1-RUNX1T1 is a heterogeneous disease entailing different prognoses. Patients with high-risk features can benefit from allogeneic hematopoietic stem cell transplantation (HSCT) or autologous HSCT. However, insufficient data about major risk factors, such as KIT mutations and measurable residual disease (MRD) status for relapse, make it difficult to clarify the benefit of each transplant strategy. Moreover, limited data are available to elucidate the exact prognostic impacts of different types of KIT mutations and optimal thresholds or time points for RUNX1-RUNX1T1 MRD assessment, particularly in the setting of HSCT. Given the lack of prospective study, the current retrospective study, including a large cohort of high-risk AML patients with RUNX1-RUNX1T1, firstly demonstrated the differentiated prognostic impact of D816V KIT mutation among various KIT mutations and clarified optimal time points and thresholds for RUNX1-RUNX1T1 MRD monitoring in the setting of HSCT.

Abstract: The prognostic significance of KIT mutations and optimal thresholds and time points of measurable residual disease (MRD) monitoring for acute myeloid leukemia (AML) with RUNX1RUNX1T1 remain controversial in the setting of hematopoietic stem cell transplantation (HSCT). We retrospectively evaluated 166 high-risk patients who underwent allogeneic (Allo-HSCT, $n=112$ ) or autologous HSCT (Auto-HSCT, $n=54$ ). D816V KIT mutation, a subtype of exon 17 mutations, was significantly associated with post-transplant relapse and poor survival, while other types of mutations in exons 17 and 8 were not associated with post-transplant relapse. Pre- and post-transplant RUNX1RUNX1T1 MRD assessments were useful for predicting post-transplant relapse and poor survival with a higher sensitivity at later time points. Survival analysis for each stratified group by D816V KIT mutation and pre-transplant RUNX1-RUNX1T1 MRD status demonstrated that Auto-HSCT was superior to Allo-HSCT in MRD-negative patients without D816V KIT mutation, while Allo-HSCT was superior to Auto-HSCT in MRD-negative patients with D816V KIT mutation. Very poor outcomes of pre-transplant MRD-positive patients with D816V KIT mutation suggested that this group should 
be treated in clinical trials. Risk stratification by both D816V KIT mutation and RUNX1-RUNX1T1 MRD status will provide a platform for decision-making or risk-adapted therapeutic approaches.

Keywords: AML; RUNX1-RUNX1T1; D816V KIT mutation; hematopoietic stem cell transplantation; measurable residual disease

\section{Introduction}

Acute myeloid leukemia (AML) with RUNX1-RUNX1T1 is known to have a favorable prognosis. However, it is also a heterogeneous disease entailing different prognoses [1-3]. Several prognostic factors, including KIT mutations and measurable residual disease (MRD) status, have been proposed [1-11]. Many types of KIT mutations have been identified in AML, and their prognostic significance has been conflicting so far [12]. Thus, the current European Leukemia Net (ELN) guideline does not support the use of KIT mutational status in clinical guidance in terms of therapeutic intervention [13]. However, recent studies have suggested different prognostic significance of each type of KIT mutations [8,9,14]. A recent prospective study has demonstrated that KIT mutations in exon 17 among three mutation hot-spots (exon 8, exon 10-11, and exon 17) are only prognostic for AML with RUNX1-RUNX1T1 through evaluation of all types of KIT mutations [8]. RUNX1-RUNX1T1 quantification by real-time quantitative polymerase chain reaction (RT-qPCR) is useful as an MRD tool for predicting relapse [15]. Some groups have suggested that RUNX1-RUNX1T1 quantification has even better predictability than KIT mutations $[1,10,16]$. However, there are several opinions regarding thresholds and time points for MRD assessment with RUNX1-RUNX1T1 [1,10,15-18]. Those optimal thresholds and time points should be evaluated according to types of KIT mutations and post-remission therapy.

Allogeneic hematopoietic stem cell transplantation (Allo-HSCT) can benefit high-risk AML patients with RUNX1-RUNX1T1 [18], although it is not generally recommended during the first complete remission (CR) [13]. A few previous reports have demonstrated that autologous hematopoietic stem cell transplantation (Auto-HSCT) had similar survival rates with Allo-HSCT [19-23]. However, those comparisons were limited by insufficient data about KIT mutations and MRD status to clarify the benefit of each transplant strategy. Therefore, we evaluated prognostic significance of different types of KIT mutations and RUNX1-RUNX1T1 quantification in high-risk AML patients with RUNX1-RUNX1T1 who underwent Allo-HSCT or Auto-HSCT to clarify the clinical relevance of each transplant strategy in each risk group. Furthermore, we elucidated optimal thresholds and time points of RUNX1-RUNX1T1 quantification during the peri-transplant period.

\section{Results}

\subsection{Patient Characteristics and KIT Mutations}

A total of 166 patients with a median age of 40 years (range, 18-69 years) underwent Allo- or Auto-HSCT in the first CR (CR1, $n=156)$ and second CR (CR2, $n=10)$. The patient-, disease-, and transplant-related characteristics according to KIT mutations are summarized in Table 1. KIT mutations were detected in 70 (42\%) of 166 patients. Among KIT exon 17 mutations, D816V mutation was identified the most frequently, followed by N822K, D816H, D816Y, and exon 8 mutations. Eighteen (26\%) of the KIT-mutated patients had multiple KIT mutations. There was no significant difference in patient- or disease-related characteristics between KIT-unmutated and KIT-mutated patients. In patients with KIT mutations, Allo-HSCT was more frequently performed than Auto-HSCT, while proportions of Allo-HSCT and Auto-HSCT were similar in patients without KIT mutations, resulting in differences of variables related to transplant procedures, including donor types, stem cell source, and transplanted CD $34^{+}$cell number. 
Table 1. Patient-, disease-, and transplant-related characteristics according to KIT mutations.

\begin{tabular}{|c|c|c|c|c|}
\hline Variables & $\begin{array}{c}\text { Overall } \\
(n=166)\end{array}$ & $\begin{array}{l}\text { KIT Unmutated } \\
\quad(n=96)\end{array}$ & $\begin{array}{l}\text { KIT Mutated } \\
\quad(n=70)\end{array}$ & $p$ \\
\hline \multicolumn{5}{|l|}{ Age at transplantation, years } \\
\hline Median (range) & $40(18-69)$ & $38(18-64)$ & $42(18-69)$ & 0.529 \\
\hline Sex, $n(\%)$ & & & & 0.814 \\
\hline Male & $105(63)$ & $60(64)$ & $45(64)$ & \\
\hline Female & $61(37)$ & $36(36)$ & $25(36)$ & \\
\hline AML type, $n(\%)$ & & & & 0.074 \\
\hline De novo & $161(97)$ & $91(95)$ & $70(100)$ & \\
\hline Therapy-related & $5(3)$ & $5(5)$ & 0 & \\
\hline \multicolumn{5}{|l|}{ WBC count per liter at diagnosis } \\
\hline Median (range) & $8.65(0.53-100.91)$ & $7.19(1.33-100.91)$ & $10.80(0.53-68.6)$ & 0.206 \\
\hline \multicolumn{5}{|c|}{ Additional cytogenetic abnormalities, $n(\%)$} \\
\hline $\operatorname{Del}(9 q)$ & $12(7)$ & $5(5)$ & $7(10)$ & 0.239 \\
\hline Trisomy 8 & $2(1)$ & $1(1)$ & $1(1)$ & 1.000 \\
\hline Loss of sex chromosome & $104(63)$ & $64(67)$ & $40(57)$ & 0.210 \\
\hline $\operatorname{Del}(7 q)$ & $3(2)$ & $1(1)$ & $2(3)$ & 0.574 \\
\hline Complex karyotype & $9(5)$ & $4(4)$ & $5(7)$ & 0.495 \\
\hline \multicolumn{5}{|l|}{ KIT mutations $n(\%)$} \\
\hline Exon 17-D816V & $29(18)$ & 0 & $29(41)$ & - \\
\hline Exon 17-D816Y & $14(8)$ & 0 & $14(20)$ & - \\
\hline Exon 17-D816H & $19(11)$ & 0 & $19(27)$ & - \\
\hline Exon $17-\mathrm{N} 822 \mathrm{~K}$ & $25(15)$ & 0 & $25(36)$ & - \\
\hline Exon 8 & $5(3)$ & 0 & $5(7)$ & - \\
\hline \multicolumn{5}{|l|}{ FLT3 mutations, $n(\%)$} \\
\hline FLT3-ITD & $9(5)$ & $6(6)$ & $3(4)$ & 0.379 \\
\hline FLT3-TKD & $3(2)$ & $1(1)$ & $2(3)$ & 0.327 \\
\hline Missing data & $9(5)$ & $7(7)$ & $2(3)$ & \\
\hline Disease status at HSCT, $n(\%)$ & & & & 0.194 \\
\hline CR1 & $156(94)$ & $88(92)$ & $68(97)$ & \\
\hline CR2 & $10(6)$ & $8(8)$ & $2(3)$ & \\
\hline Donor type, $n(\%)$ & & & & 0.001 \\
\hline Matched sibling & $64(39)$ & $34(35)$ & $30(43)$ & \\
\hline Matched unrelated & $25(15)$ & $10(10)$ & $15(21)$ & \\
\hline Haploidentical & $23(14)$ & $9(9)$ & $14(20)$ & \\
\hline Autologous & $54(32)$ & $43(45)$ & $11(16)$ & \\
\hline Stem cell source, $n(\%)$ & & & & 0.003 \\
\hline Peripheral blood & $111(67)$ & $55(57)$ & $56(80)$ & \\
\hline Bone marrow & $28(17)$ & $18(19)$ & $10(14)$ & \\
\hline Peripheral blood and bone marrow & $27(16)$ & $23(24)$ & $4(6)$ & \\
\hline Conditioning intensity, $n(\%)$ & & & & 0.256 \\
\hline Myeloablative & $105(63)$ & $57(59)$ & $48(69)$ & \\
\hline Reduced intensity & $61(37)$ & $39(41)$ & $22(31)$ & \\
\hline \multicolumn{5}{|c|}{ Interval from diagnosis to transplant, days } \\
\hline Median (range) & $194(96-260)$ & $195(96-260)$ & $184(102-243)$ & 0.174 \\
\hline \multicolumn{5}{|l|}{$\mathrm{CD} 34^{+}$cells $\times 10^{6} / \mathrm{kg}$ in graft } \\
\hline Median (range) & $3.88(0.73-16.73)$ & $3.52(1.01-16.10)$ & $4.88(0.73-16.73)$ & 0.059 \\
\hline
\end{tabular}

Abbreviations: AML, acute myeloid leukemia; CR1, first complete remission; CR2, second complete remission; HSCT, hematopoietic stem cell transplantation; $n$, number; WBC, white blood cells.

\subsection{Impact of KIT Mutations Status on RUNX1-RUNX1T1 MRD Kinetics and Survival Outcomes \\ Impacts of KIT mutations on RUNX1-RUNX1T1 MRD kinetics were evaluated (Table 2). RUNX1-RUNX1T1 levels were continuously decreased after chemotherapies and transplan- tation. Mean bone marrow (BM) $\log _{10}$-transformed transcript levels at post-induction, pre- HSCT, and 1 month after HSCT were significantly higher and degrees of log reduction were significantly lower in KIT-mutated patients than KIT-unmutated patients. Patients with D816V KIT mutation had significantly less reduction in RUNX1-RUNX1T1 level compared to patients without D816V KIT mutation. For the other hot mutations, including D816Y,}


D816H, N822K, or exon 8 mutations, there was no significant difference in RUNX1-RUNX1T1 MRD kinetics between patients with and without such mutations (data not shown). These data suggest that KIT mutations, particularly D816V KIT mutation, have some resistance to chemotherapies and/or transplantation.

Table 2. Impact of KIT mutations on kinetics of RUNX1-RUNX1T1 transcript levels *

\begin{tabular}{|c|c|c|c|c|c|c|c|}
\hline \multirow{2}{*}{ Variables } & \multirow{2}{*}{$\begin{array}{c}n \\
\text { Unmutated } \\
\text { vs. Mutated }\end{array}$} & \multicolumn{3}{|c|}{$\log _{10}$ Transformed Transcript Levels } & \multicolumn{3}{|c|}{ Log Reduction } \\
\hline & & Unmutated & Mutated & $p$ & Unmutated & Mutated & $p$ \\
\hline \multicolumn{8}{|l|}{ KIT mutations } \\
\hline Diagnosis & 96 vs. 70 & $0.68 \pm 0.02$ & $0.64 \pm 0.03$ & 0.295 & - & - & - \\
\hline Post-induction & 87 vs. 62 & $-2.39 \pm 0.11$ & $-2.02 \pm 0.11$ & 0.021 & $-3.07 \pm 0.10$ & $-2.65 \pm 0.12$ & 0.009 \\
\hline Pre-HSCT & 96 vs. 70 & $-4.02 \pm 0.14$ & $-3.32 \pm 0.17$ & 0.002 & $-4.70 \pm 0.14$ & $-3.95 \pm 0.17$ & 0.001 \\
\hline 1 month after HSCT & 43 vs. 40 & $-4.69 \pm 0.21$ & $-4.05 \pm 0.24$ & 0.044 & $-5.38 \pm 0.21$ & $-4.65 \pm 0.24$ & 0.024 \\
\hline 3 months after HSCT & 57 vs. 45 & $-5.07 \pm 0.15$ & $-4.63 \pm 0.26$ & 0.140 & $-5.78 \pm 0.15$ & $-5.24 \pm 0.26$ & 0.076 \\
\hline \multicolumn{8}{|l|}{ D816V KIT mutation } \\
\hline Diagnosis & 137 vs. 29 & $0.67 \pm 0.02$ & $0.61 \pm 0.04$ & 0.160 & - & - & - \\
\hline Post-induction & 122 vs. 27 & $-2.30 \pm 0.09$ & $-1.92 \pm 0.17$ & 0.068 & $-2.97 \pm 0.09$ & $-2.52 \pm 0.17$ & 0.026 \\
\hline Pre-HSCT & 137 vs. 29 & $-3.82 \pm 0.12$ & $-3.26 \pm 0.25$ & 0.055 & $-4.50 \pm 0.12$ & $-3.86 \pm 0.25$ & 0.031 \\
\hline 1 month after HSCT & 67 vs. 16 & $-4.55 \pm 0.17$ & $-3.68 \pm 0.38$ & 0.032 & $-5.21 \pm 0.17$ & $-4.25 \pm 0.41$ & 0.019 \\
\hline 3 months after HSCT & 85 vs. 17 & $-5.00 \pm 0.14$ & $-4.25 \pm 0.44$ & 0.049 & $-5.68 \pm 0.14$ & $-4.85 \pm 0.46$ & 0.029 \\
\hline
\end{tabular}

Abbreviations: HSCT, hematopoietic stem cell transplantation; $n$, number; ${ }^{*}$ RUNX1-RUNX1T1 transcript levels were normalized with respect to the number of $A B L 1$ transcripts and expressed as copy numbers per $10^{5}$ copies of $A B L 1$. Data were expressed as mean \pm SEM.

At a median follow-up of 60 months (range, 6-131 months), 21 patients relapsed at a median of 8 months (range, 4-25 months) after HSCT. A higher trend of cumulative incidence of relapse (CIR) in KIT-mutated patients was observed (Figure 1A and Supplementary Table S2). Multivariate analysis with an adjustment relating to disease status at transplant revealed significant associations between KIT mutations and CIR (Supplementary Table S3). However, a significant difference of CIR was only observed in the group with Auto-HSCT, whereas there was no significant difference of CIR in the group with Allo-HSCT (Figure 1B,C). In a subgroup analysis according to types of KIT mutations, patients with D816V KIT mutation had significantly increased CIR compared to patients with other KIT mutations or without KIT mutations, while other types of mutations in exon 17 or 8 were not associated with post-transplant relapse (Supplementary Figure S2 and Supplementary Table S2). Moreover, patients with D816V KIT mutation had significantly higher CIR in both Allo-HSCT and Auto-HSCT groups than patients without such mutation (Figure 1D-F), which translated into inferior disease-free survival (DFS) and overall survival (OS, Supplementary Figure S3 and Supplementary Table S3).

\subsection{Optimal Time Points and Thresholds for RUNX1-RUNX1T1 MRD Monitoring}

Results of serial RT-qPCR assays of RUNX1-RUNX1T1 according to transplant type are presented in Supplementary Table S4. Both Allo-HSCT and Auto-HSCT significantly decreased RUNX1-RUNX1T1 levels after transplant (Figure 2A). RUNX1-RUNX1T1 levels at pre-HSCT were significantly higher in the group of Allo-HSCT, which had more D816V KIT-mutated patients, than in the group of Auto-HSCT. Such difference persisted at 1 month after transplantation. Their levels became similar at 3 months after transplantation (Figure 2A and Supplementary Table S4). Relapsed patients had significantly higher RUNX1-RUNX1T1 levels at pre-HSCT and at 1 or 3 months after HSCT than non-relapsed patients. Such levels in relapsed patients were not significantly decreased after HSCT compared to the progressive decrease in non-relapsed patients (Figure 2B). Receiver operating characteristic (ROC) curve analysis revealed that RUNX1-RUNX1T1 levels at all time points, including pre-HSCT and 1 or 3 months after HSCT, could predict post-transplant relapse (Figure $2 \mathrm{C}$ ). The sensitivity and specificity at each time point (Supplementary Table S5) showed a trend of improvement as time went by. 
A

$$
\text { All patients }
$$

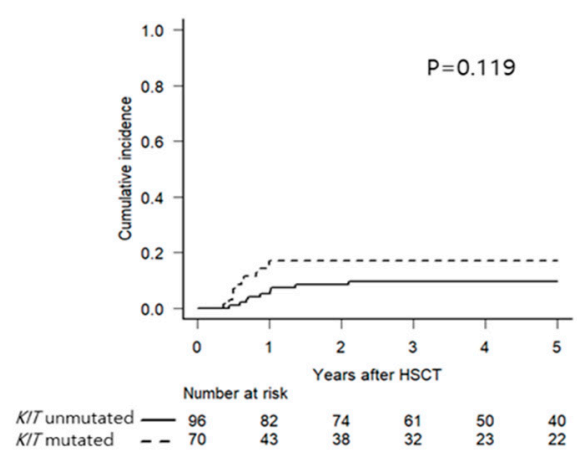

B

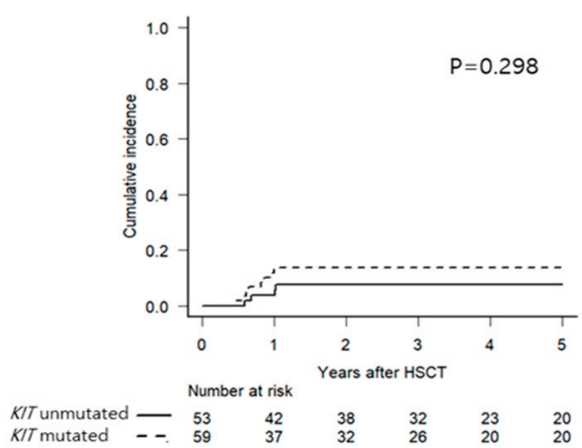

C

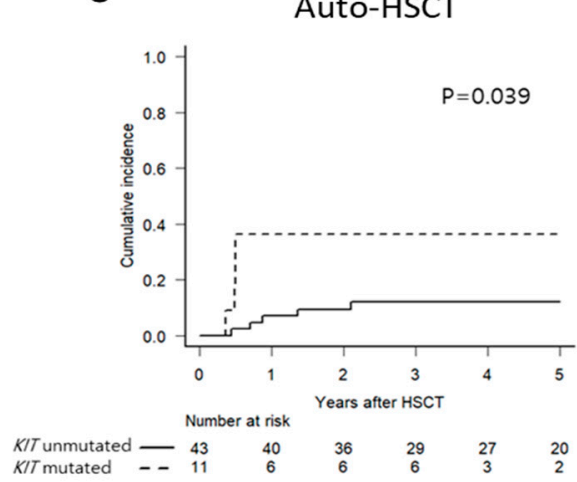

D

All patients

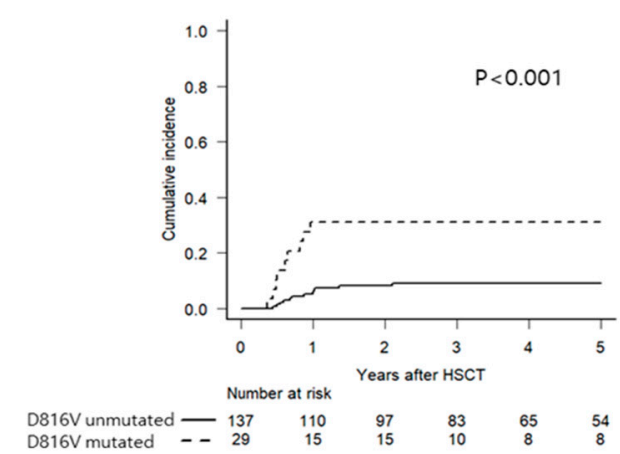

$E$

Allo-HSCT

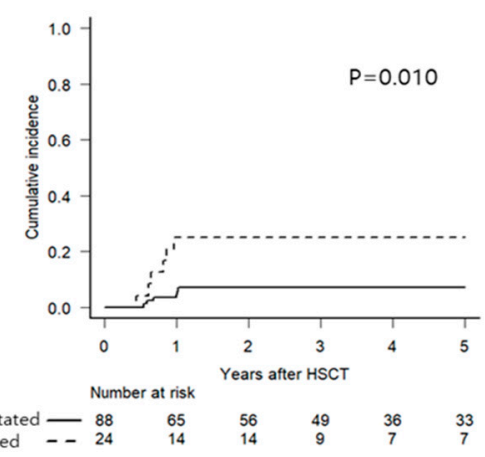

$\mathrm{F}$

Auto-HSCT

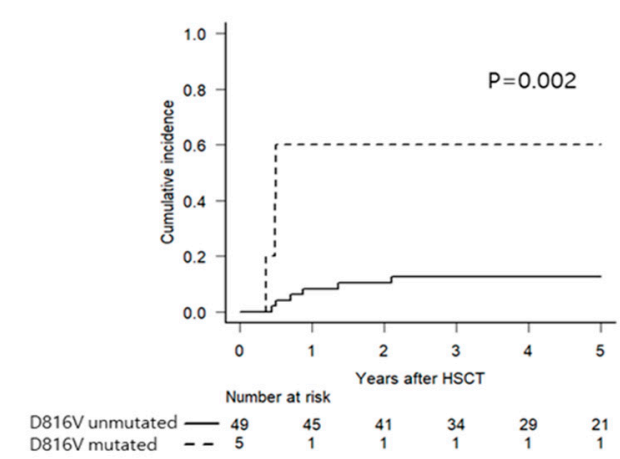

Figure 1. Cumulative incidence of relapse according to KIT mutations (A-C) or D816V KIT mutation (D-F) in all patients (A,D) or patients in Allo-HSCT (B,E) and Auto-HSCT (C,F) groups.

To elucidate optimal thresholds of RUNX1-RUNX1T1 levels for predicting posttransplant relapse, various cutoffs, including copy numbers (1000 copies, 500 copies, 250 copies, 100 copies, 50 copies, 10 copies, and 0 copy) and degrees of log reduction (3 log or $4 \mathrm{log}$ ) at each time point, were compared (Supplementary Figure S4 and Supplementary Table S5). Every cutoff level was useful for identifying patients at high risk or relapse. A $3 \log$ reduction at each time point appeared to be the most effective one based on sensitivity and specificity (pre-HSCT, 38\% and 91\%; 1 month after HSCT, 75\% and $89 \%$; 3 months after HSCT, $83 \%$ and 94\%; Supplementary Table S5). Multivariate analysis of significant factors found in univariate analysis (Supplementary Table S2) revealed that MRD positivity defined by $3 \log$ reduction at each time point independently predicted CIR, which translated into inferior DFS and OS (Supplementary Table S6). Figure 3 and 
Supplementary Figure S5 show survival outcomes according to MRD positivity defined by $3 \log$ reduction.

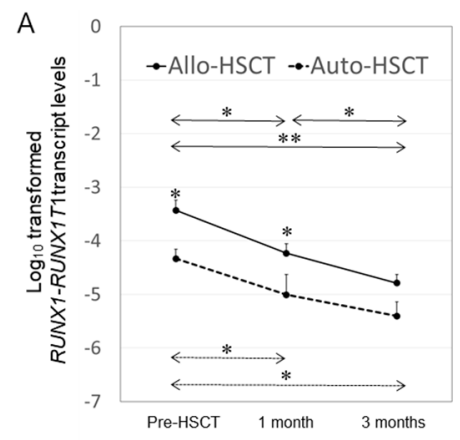

C

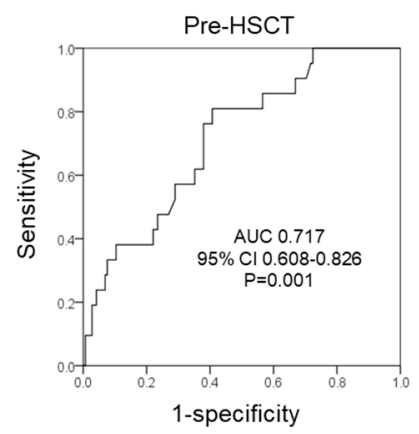

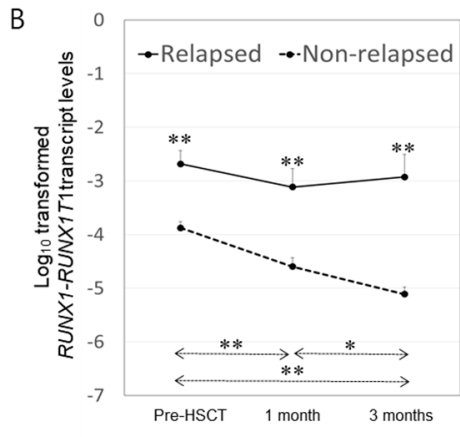

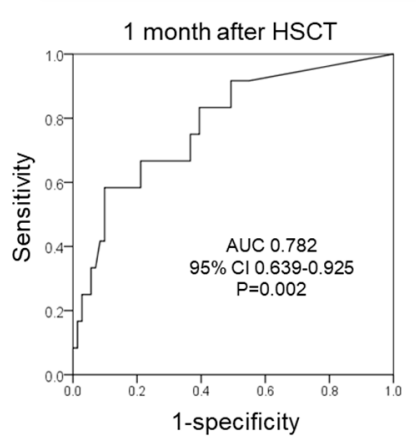

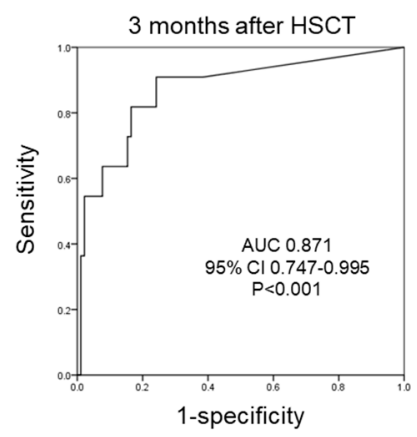

Figure 2. RUNX1-RUNX1T1 expression kinetics during peri-transplant period and optimal time points. (A) RUNX1RUNX1T1 expression kinetics according to transplant type (Allo-HSCT vs. Auto-HSCT) before HSCT and at 1 or 3 months after HSCT (numbers of Allo-HSCT vs. Auto-HSCT; before, 112 vs. 54; 1 month, 70 vs. 13; 3 months, 86 vs. 16). (B) RUNX1RUNX1T1 expression kinetics according to the occurrence of relapse (numbers of relapsed patients vs. non-relapsed patients; before, 21 vs. 145; 1 month, 12 vs. 71; 3 months, 11 vs. 91). (C) Receiver operating characteristic (ROC) curve analysis of RUNX1-RUNX1T1 levels at each time point. RUNX1-RUNX1T1 levels were normalized to the number of $A B L 1$ transcripts and expressed as copy numbers per $10^{5}$ copies of $A B L 1$. Results are expressed as mean \pm SEM. ${ }^{*} p<0.05,{ }^{* *} p<0.01$. AUC indicates area under curve.

\subsection{Prognostic Independency of D816V KIT Mutation and RUNX1-RUNX1T1 MRD Status}

Multivariate analysis was performed to compare prognostic significance for predicting post-transplant relapse among three major factors found in univariate analysis (Supplementary Table S2): disease status at transplant, D816V KIT mutation, and RUNX1RUNX1T1 MRD status defined by 3 log reduction (Model \#2 in Table 3). It revealed that MRD status at each time point and disease status at transplant were significant for predicting post-transplant relapse. D816V KIT mutation remained significant in the multivariate model. We also evaluated prognostic independency of KIT mutations shown in Supplementary Table S3 and RUNX1-RUNX1T1 MRD status (Model \#1 in Table 3). In contrast to D816V KIT mutation, KIT mutations including all types lost their significance in the multivariate model.

\subsection{Outcomes of Transplant Type in Each Group Stratified by D816V KIT Mutation and RUNX1-RUNX1T1 MRD Status at Pre-HSCT}

Univariate analysis showed that the Auto-HSCT group had favorable DFS and OS due to both reduced non-relapse mortality (NRM) rates and similar CIR compared to Allo-HSCT group (Supplementary Figure S6 and Supplementary Table S2). However, multivariate models (Supplementary Tables S3 and S6) revealed no significant difference in DFS or OS between Allo- and Auto-HSCT groups, which might be due to significant differences in patient- and/or disease-related characteristics such as older age, more CR2, and higher 
number of white blood cell (WBC) counts at diagnosis and transplanted CD34+ cells in the Allo-HSCT group than Auto-HSCT group (Supplementary Table S1). In particular, proportions of D816V KIT mutation and RUNX1-RUNX1T1 levels at pre-HSCT, which were demonstrated as important factors associated with post-transplant relapse, were significantly higher in the Allo-HSCT group than in the Auto-HSCT group.

Relapse

A

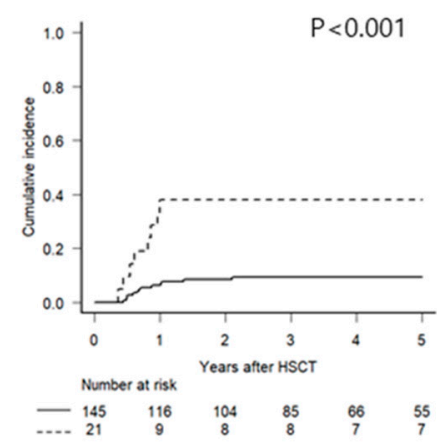

B

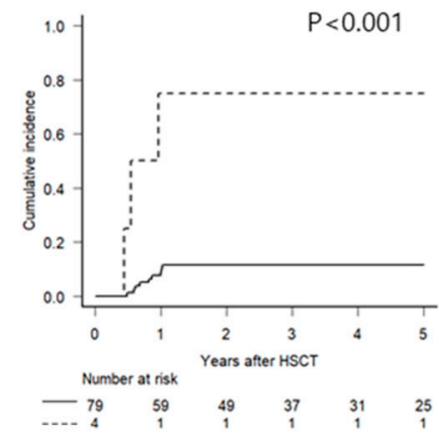

C

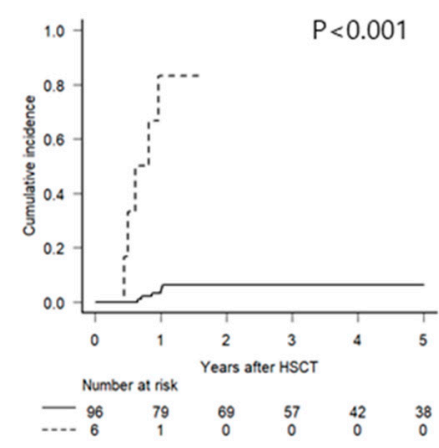

Overall survival

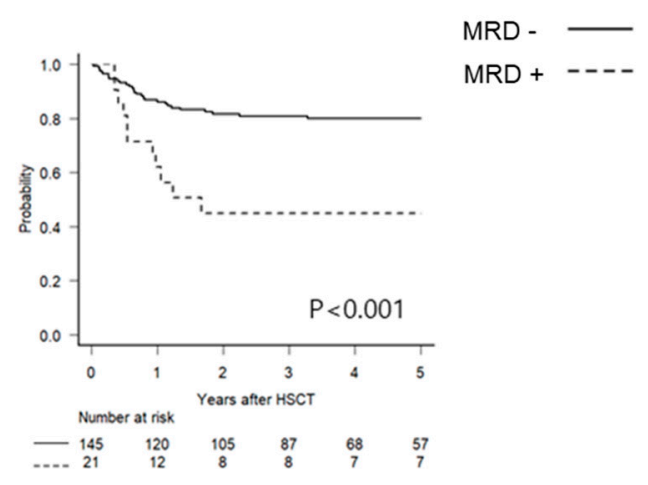

Figure 3. Survival outcomes according to MRD positivity defined by $3 \log$ reduction in RUNX1RUNX1T1 levels at pre-HSCT (A) and at 1 month (B) or 3 months (C) after HSCT.

Thus, we stratified patients by D816V KIT mutation and MRD positivity defined by $3 \log$ reduction in RUNX1-RUNX1T1 at pre-HSCT into four groups to elucidate effects of transplant type in each risk group. Patients with both D816V KIT mutation and MRD positivity had significantly increased CIR compared to those in the other three groups (Figure 4A). In subgroup analyses for effects of transplant type in each group (Figure 4B-E and Supplementary Figure S7), among MRD-negative patients, Allo-HSCT was more beneficial to prevent relapse in patients with D816V KIT mutation than Auto-HSCT, resulting in superior DFS and OS for patients in the Allo-HSCT group. In contrast, superior DFS and OS of Auto-HSCT to Allo-HSCT were observed in patients without D816V KIT mutation due to improved NRM in Auto-HSCT without significant difference in CIR. Among 
MRD-positive patients, effects of transplant type were not clear due to the small number of patients in each group. However, we observed that patients with D816V KIT mutation had significantly higher risk for relapse than patients without such mutation, which translated into very poor DFS and OS. NRM rather than CIR was a determinant factor for survival in MRD-positive patients without D816V KIT mutation who underwent Allo-HSCT.

Table 3. Multivariate analyses to evaluate the prognostic independency of D816V KIT mutation and RUNX1-RUNX1T1 MRD status at each time point for predicting post-transplant relapse.

\begin{tabular}{|c|c|c|c|c|c|c|}
\hline \multirow{2}{*}{ Model \#1 } & \multicolumn{2}{|c|}{ Pre-HSCT } & \multicolumn{2}{|c|}{1 Month after HSCT } & \multicolumn{2}{|c|}{3 Months after HSCT } \\
\hline & HR (95\% CI) & $p$ Value & HR $(95 \%$ CI) & $p$ Value & HR $(95 \%$ CI $)$ & $p$ Value \\
\hline \multicolumn{7}{|c|}{ RUNX1-RUNX1T1 levels } \\
\hline$\geq 3 \log$ reduction & 1 & & 1 & & 1 & \\
\hline$<3 \log$ reduction & $5.31(2.06-13.65)$ & 0.001 & $7.15(1.59-32.11)$ & 0.010 & $22.23(5.03-98.23)$ & $<0.001$ \\
\hline \multicolumn{7}{|l|}{ KIT mutations } \\
\hline Unmutated & 1 & & 1 & & 1 & \\
\hline Mutated & $2.14(0.81-5.62)$ & 0.123 & $4.07(0.73-22.72)$ & 0.110 & $2.69(0.42-17.46)$ & 0.299 \\
\hline \multicolumn{7}{|l|}{ Disease state } \\
\hline CR1 & 1 & & 1 & & 1 & \\
\hline CR2 & $7.55(2.0-28.47)$ & 0.003 & $13.3(2.18-81.41)$ & 0.005 & $9.37(1.31-66.87)$ & 0.026 \\
\hline \multirow{2}{*}{ Model \#2 } & \multicolumn{2}{|c|}{ Relapse } & \multicolumn{2}{|c|}{1 Month after HSCT } & \multicolumn{2}{|c|}{3 Months after HSCT } \\
\hline & HR $(95 \%$ CI) & $p$ Value & HR $(95 \%$ CI) & $p$ Value & HR $(95 \%$ CI $)$ & $p$ Value \\
\hline \multicolumn{7}{|c|}{ RUNX1-RUNX1T1 levels } \\
\hline$\geq 3 \log$ reduction & 1 & & 1 & & 1 & \\
\hline$<3 \log$ reduction & 4.89 (1.91-12.49) & 0.001 & $4.76(0.95-23.86)$ & 0.058 & $20.50(4.68-89.81)$ & $<0.001$ \\
\hline \multicolumn{7}{|l|}{ D816V KIT mutation } \\
\hline Unmutated & 1 & & 1 & & 1 & \\
\hline Mutated & $4.10(1.63-10.30)$ & 0.003 & $4.56(1.02-20.33)$ & 0.047 & $4.33(1.01-18.56)$ & 0.049 \\
\hline \multicolumn{7}{|l|}{ Disease state } \\
\hline CR1 & 1 & & 1 & & 1 & \\
\hline CR2 & $6.75(1.86-24.5)$ & 0.004 & $9.22(2.04-41.66)$ & 0.004 & 7.59 (1.31-44.20) & 0.024 \\
\hline
\end{tabular}

Abbreviations: Allo-HSCT, allogeneic HSCT; Auto-HSCT, autologous HSCT; CI, confidence interval; CR1, first complete remission; CR2, second complete remission; HR, hazard ratio; HSCT, hematopoietic stem cell transplantation; $n$, number.

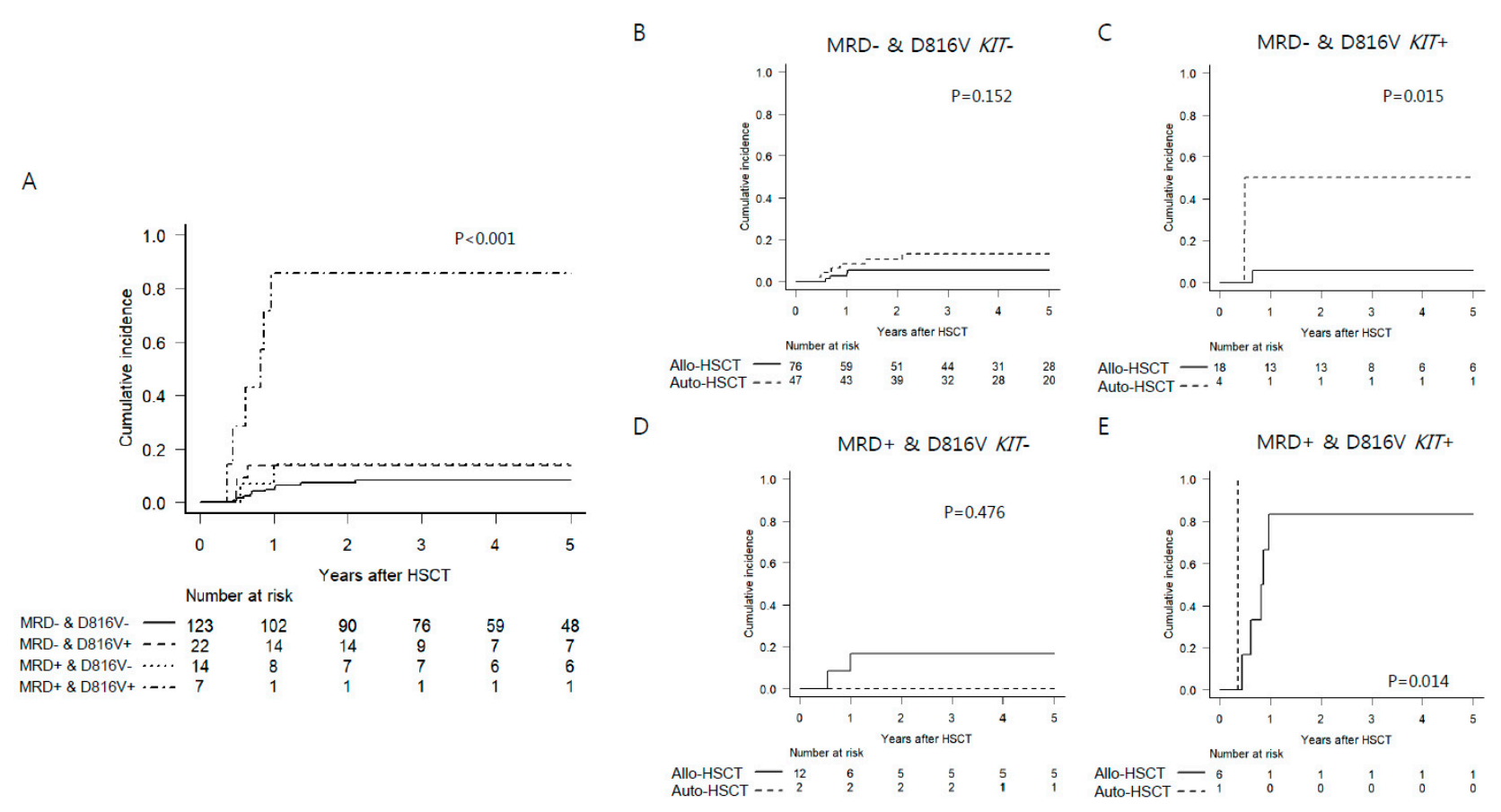

Figure 4. Cumulative incidence of relapse (CIR) according to both D816V KIT mutation and MRD positivity defined by 3 log reduction in RUNX1-RUNX1T1 levels at pre-HSCT (A) and effects of transplant types on CIR in each stratified group (B-E). 


\section{Discussion}

Allo-HSCT is not generally considered during CR1 for AML with RUNX1-RUNX1T1 [13]. However, patients with high-risk features can benefit from Allo-HSCT [18], and recent data suggest that Auto-HSCT may be an alternative [19-23]. Nevertheless, insufficient data about major risk factors, such as KIT mutations and MRD status for relapse, make it difficult to clarify the benefit of each transplant strategy. Moreover, limited data are available to elucidate prognostic impacts of different types of KIT mutations and optimal thresholds or time points for RUNX1-RUNX1T1 MRD assessment, particularly in the setting of HSCT. The current study, including high-risk AML patients with RUNX1-RUNX1T1 who underwent Allo- and AutoHSCT. demonstrated that both D816V KIT mutation and peri-transplant RUNX1-RUNX1T1 MRD monitoring were independently associated with post-transplant relapse and survival. Of note, in analyses of stratified groups according to the presence of D816V KIT mutation and pre-transplant MRD status, Allo-HSCT and Auto-HSCT were superior to each other in MRD-negative patients with and without D816V KIT mutation, respectively. In addition, poor outcomes in pre-transplant MRD-positive patients with D816V KIT mutation due to increased post-transplant relapse suggest the necessity of pre- or post-transplant therapeutic targeting with small molecules against the RUNX1-RUNX1T1 protein, the use of tyrosine kinase inhibitors (dasatinib and FLT3 inhibitors), epigenetic modulators [24], and cellular approaches $[25,26]$ for this group of patients.

Prognostic impacts of KIT mutations in core-binding factor AML, including RUNX1RUNX1T1 and CBFB-MYH11, have been controversial [12]. Thus, they are not included in guidelines for risk stratification in the current ELN guideline [13]. However, recent studies have reported the prognostic importance of KIT mutations in AML with RUNX1RUNX1T1, but not in those with CBFB-MYH11 [8,23]. Prognostic impacts of each type of KIT mutations may be different $[8,9,14]$. Compared to other KIT mutations, exons 17 and/or 8 mutations are more likely to adversely affect survival $[8,9,14]$. The GermanAustrian AML Study Group (AMLSG) has demonstrated correlations of RUNX1-RUNX1T1 levels after chemotherapies with KIT mutations (exons 17 and/or 8), with KIT-unmutated patients achieving deeper MRD reductions at the end of treatment [10]. This indicates that KIT-mutated patients have some resistance to chemotherapies. A recent prospective study including patients treated with chemotherapy only has demonstrated that KIT mutations in exon 17 are prognostic factor in AML with RUNX1/RUNX1T1 after evaluating all types of KIT mutations [8]. In our unique cohort of transplanted high-risk patients, we firstly showed that D816V KIT mutation, a subtype of exon 17 mutations, rather than all KIT mutations, was significantly associated post-transplant relapse and poor survival. Indeed, other types of mutations in exons 17 (D816H, D816Y, or N822K) and 8 showed no significant association with increased risk for relapse. Less reduction in RUNX1-RUNX1T1 level in D816V KIT-mutated patients after not only chemotherapy, in line with AMLSG, but also HSCT, supports poor prognostic features related with relapse. A recent study by Tarlock et al. investigated the functional impact of distinct mutation subsets of KIT mutations in an in vitro model [27]. They demonstrated that the D816V mutation resulted in more potent KIT phosphorylation as well as increased detection of immature vs. mature form of KIT compared with N822K or exon 8 mutations, which supports the distinctive prognostic role of D816V KIT mutation in our study. Some reports have suggested that the prognostic impacts of KIT mutations were outweighed by RUNX1-RUNX1T1 level during treatment in the setting of chemotherapy $[1,10]$ or HSCT [16]. In contrast, our results showed that D816V KIT mutation remained a significant factor for post-transplant relapse with RUNX1-RUNX1T1 MRD status in multivariate models, suggesting its independent prognostic power, which needs to be further evaluated in a larger cohort. The prognostic importance of KIT mutation in specific loci in the current study, as well as in other recent reports $[8,9,14]$, provides evidences that the current ELN guidelines need to be revised to include KIT mutational status for risk stratification.

Several studies have demonstrated that the persistent presence of RUNX1-RUNX1T1 transcript is a strong predictor of relapse, while optimal time points or thresholds during 
the active treatment phase remain controversial [15]. Recently, AMLSG has proposed a refined practical guidance for RUNX1-RUNX1T1 MRD monitoring, emphasizing the achievement of MRD negativity in both BM and PB after completion of therapy [10], in contrast to suggested importance of $>3 \log$ reduction in $\mathrm{BM}$ between diagnosis and the end of induction [13] or consolidation [28] in previous studies. However, few data are available about whether RUNX1-RUNX1T1 MRD monitoring can continue to serve as an efficient tool for risk stratification after HSCT. An earlier study from China has suggested that not pre-transplant but post-transplant RUNX1-RUNX1T1 MRD monitoring with a cutoff of $3 \log$ reduction could discriminate patients at high risk of post-transplant relapse, and post-transplant RUNX1-RUNX1T1 MRD monitoring was also more predictive of relapse risk than KIT mutations [16]. In contrast, our data with a larger cohort revealed that pre- and post-transplant RUNX1-RUNX1T1 MRD monitoring were useful for predicting post-transplant relapse. They were more predictive than whole KIT mutations, similar to data from China. However, they had similar predictive power to the D816V KIT mutation. Furthermore, based on sensitivity and specificity at pre-HSCT and at 1 or 3 months after HSCT, our results clearly demonstrated that the optimal threshold was not an achievement of MRD negativity, but $3 \log$ reductions in the setting of HSCT. The sensitivity was greater at 3 months after HSCT, whereas the specificity was similar at all time points. Thus, the optimal time point for RUNX1-RUNX1T1 MRD monitoring might be 3 months after HSCT, which would be available to apply additional therapies in an attempt to prevent relapse because all relapse events were observed beyond 4 months after HSCT. On the other hand, earlier recognition of high-risk patients at pre-HSCT would be helpful for applying additional cellular approaches to enhance anti-leukemia effects, which needs considerable time for preparation $[25,26]$. Further studies are warranted to validate the role of risk-adapted approaches based on RUNX1-RUNX1T1 MRD monitoring as well as KIT mutations.

The current study evaluated a large cohort of high-risk AML patients with RUNX1RUNX1T1 who underwent Allo-HSCT or Auto-HSCT to clarify the prognostic significance of different types of KIT mutations and RUNX1/RUNX1T1 quantification. A limitation of our study was that we focused on KIT mutations of exons 17 and 8 . These mutations have been reported to adversely affect survival of AML patients with RUNX1-RUNX1T1 [8,9,14]. Interactions of KIT mutations with other concurrent mutations, such as ASXL1 [14] or the recently identified SMC1A and DHX15 [29], need to be further evaluated. Differences in pre-transplant characteristics, particularly RUNX1/RUNX1T1 level and presence of KIT mutations, between Allo- and Auto-HSCT due to the retrospective nature of this study and transplant decisions based on donor availability should be considered when interpreting our data. That is why we stratified all patients into four groups based on both RUNX1/RUNX1T1 levels and D816V KIT mutation, and compared Allo-HSCT with AutoHSCT in each group. Different regimens for induction, including gemtuzumab ozogamicin in patients with CD33 or dasatinib in patients with KIT mutations, or consolidation, such as high-dose cytarabine, may affect RUNX1-RUNX1T1 MRD levels and outcomes [30]. Thus, the combined impact of KIT mutations and RUNX1-RUNX1T1 MRD status should be further evaluated in patients treated with those regimens without transplantation.

\section{Materials and Methods}

\subsection{Patients}

We retrospectively evaluated 183 consecutive AML patients with RUNX1/RUNX1T1 in remission who underwent HSCT at the Catholic Hematology Hospital between 2009 and 2018. After excluding nine patients who underwent second HSCT and eight patients without data of KIT mutations $(n=3)$ or pre-transplant RUNX1/RUNX1T1 MRD data $(n=5), 166$ patients were finally included in this study (Supplementary Figure S1). Before transplant, 141 of 166 patients were classified as high-risk AML by the persistence of RUNX1/RUNX1T1 transcript ( $n=130,78 \%$ ) and/or KIT mutations ( $n=70,42 \%)$. Twenty patients had other high-risk features such as loss of $Y$ chromosome $(n=17)[3,31]$, second 
CR $(n=2)$, and extramedullary disease $(n=1)$ [32], while five patients persisted to undergo HSCT. All patients were treated with intensive induction and one or two cycles of consolidation chemotherapy [33]. Patients underwent Allo-HSCT if an available donor was found during consolidation. If the patient did not have an available donor, patients underwent Auto-HSCT. For Auto-HSCT, CD $34^{+}$stem cells were collected for three days after neutrophil count recovered from consolidation chemotherapy [34]. The Institutional Review Board of the Catholic Medical Center approved the current study (\#KC16TISI0438). All analyses were performed according to the Institutional Review Board guidelines and tenets of the Declaration of Helsinki.

\subsection{Chemotherapy and Transplant Procedures}

All patients were treated according to our standard protocol, consisting of " $3+7$ " idarubicin (IDA, $12 \mathrm{mg} / \mathrm{m}^{2}$, intravenous infusion) plus cytarabine (Ara-C, $100 \mathrm{mg} / \mathrm{m}^{2}$ continuously infused for $24 \mathrm{~h}$ ) for remission-induction chemotherapy. After achieving CR, two consolidation chemotherapies were administered. Consolidation chemotherapies consisted of " $3+5$ " mitoxantrone (12 mg/m², intravenous infusion) or IDA (12 mg $/ \mathrm{m}^{2}$, intravenous infusion) plus an intermediate dose of Ara-C $\left(1.0 \mathrm{~g} / \mathrm{m}^{2}\right.$, intravenous infusion, bid), which were alternated. Transplant-related characteristics of the patients according to transplant type are listed in Supplementary Table S1. For transplants from matched sibling or unrelated donors, myeloablative or reduced-intensity conditioning regimens consisting of fludarabine $\left(150 \mathrm{mg} / \mathrm{m}^{2}\right)$ and busulfex $(6.4 \mathrm{mg} / \mathrm{kg})$, with or without fractionated total body irradiation (TBI) of $400 \mathrm{cGy}$, were used based on age and/or comorbidity [35]. For transplants from haploidentical donors, all patients received a reduced-intensity toxicity conditioning regimen consisting of fludarabine $\left(150 \mathrm{mg} / \mathrm{m}^{2}\right)$, busulfex $(6.4 \mathrm{mg} / \mathrm{kg})$, and fractionated TBI (800 cGy) [35]. For Auto-HSCT, an myeloablative regimen consisting of TBI (1200 cGy), cytarabine $\left(9 \mathrm{~g} / \mathrm{m}^{2}\right)$, and melphalan $\left(100 \mathrm{mg} / \mathrm{m}^{2}\right)$ was used for the majority (91\%) of patients [35]. Graft-versus-host disease prophylaxis was performed using a short-course methotrexate plus cyclosporine for transplants from matched sibling donors or tacrolimus for transplants from matched unrelated and haploidentical donors. Anti-thymocyte globulin (Sanofi/Genzyme, Cambridge, MA, USA) was given at a fixed dose of $5.0 \mathrm{mg} / \mathrm{kg}$ for transplants from haploidentical donors, while different doses $(1.25-5.0 \mathrm{mg} / \mathrm{kg}$ ) were used over different time periods for transplants from matched unrelated donors. Other general transplant-related procedures were performed as previously described [34].

\subsection{Cytogenetic and Molecular Analyses}

For detection of karyotypes, BM was used. At least 20 metaphases were analyzed with the Giemsa banding method after 24 or $48 \mathrm{~h}$ of unsynchronized culture. The presence of RUNX1/RUNX1T1 was confirmed by a multiplex reverse transcriptase polymerase chain reaction screening assay using a HemaVision Kit (DNA Technology, Risskov, Denmark). Mutations in exons 8 and 17 of the KIT gene were analyzed as previously reported [35]. Screening tests for detecting KIT mutations (D816V, D816H, D816Y and N822K) in exon 17 were performed with an allele-specific RT-qPCR assay (Real-Q KIT Screening Kit; BioSewoom, Seoul, Korea). Positive samples were genotyped using a Real-Q KIT Genotyping Kit (BioSewoom) for discriminating each mutation. Detection of KIT mutations in exon 8 was performed by direct sequencing [36]. Mutations in FLT3, NPM1, and CEBPA were also analyzed using protocols established since 2008 [34,36].

\subsection{RUNX1-RUNX1T1 MRD Assessment and Definitions}

For the assessment of MRD, BM samples at pre-HSCT and at 1 or 3 months after HSCT were used for RT-qPCR for RUNX1-RUNX1T1. Total RNA was isolated from patients' BM aspirates using the High Pure RNA Isolation Kit (Roche Diagnostics, Mannheim, Germany). Nucleic acid quality and quantity was measured using a NanoDrop 1000 spectrophotometer (Thermo Fisher Scientific, Waltham, MA, USA). Reverse transcription was carried out 
using Transcriptor First Strand cDNA Synthesis Kit (Roche Applied Science, Mannheim, Germany). RT-qPCR assay was performed using the Real- ${ }^{\text {TM }}$ RUNX1-RUNX1T1 Quantification kit (Bioseum, Seoul, Korea) according to the manufacturer's instruction. The kit consisted of RUNX1-RUNX1T1 and ABL1 standard materials of four for each concentration. After preparing PCR mixture for RUNX1-RUNX1T1 and ABL1 (4 $\mu \mathrm{L}$ of each probe and primer mixture, $4 \mu \mathrm{L}$ of cDNA or standard material, $12.5 \mu \mathrm{L}$ of PCR reaction mixture, and $4.5 \mu \mathrm{L}$ of distilled water), reactions were performed using an ABI 7500 Real-Time PCR system (Applied Biosystems, Foster City, CA, USA). PCR conditions were 2 min at $50{ }^{\circ} \mathrm{C}$, $10 \mathrm{~min}$ at $95^{\circ} \mathrm{C}, 45$ cycles of $15 \mathrm{~s}$ at $95^{\circ} \mathrm{C}$ and $1 \mathrm{~min}$ at $60^{\circ} \mathrm{C}$. RUNX1-RUNX1T1 fusion genes calculated with standard materials were normalized with respect to the number of ABL1 transcripts and expressed as copy numbers per $1 \times 10^{5}$ copy of ABL1. Assays were performed in replicate with appropriate controls. We tested different thresholds for levels of RUNX1-RUNX1T1 or log reduction compared to levels at diagnosis. ROC curve analysis was used to determinate optimal time points for MRD assessment with RUNX1-RUNX1T1. Please refer to supplementary Methods for detailed descriptions for quality control of the MRD assessment.

\subsection{Statistical Analysis}

The categorical variables were compared using the Chi-square test or Fisher's exact test while the continuous variables were analyzed with the Student's t-test or Wilcoxon's rank-sum test. OS and DFS curves were plotted using the Kaplan-Meier method and analyzed with the log-rank test. The cumulative incidence was used to estimate the probability of the CIR and NRM. Non-relapse death and relapse were treated as competing risk factors for CIR and NRM, respectively, and compared using the Gray test. Multivariate analysis included variables with $p$-values of $<0.10$, as determined by univariate analysis, and were considered for entry into the model selection procedure based on the Cox proportional hazards model or a proportional hazards model for the sub-distribution of the competing risk factors. Statistical significance was indicated by a $p$-value of less than or equal to 0.05 (two-tailed). All statistical analyses were conducted using SPSS, version 13.0 (SPSS, Inc., Chicago, IL, USA) and R-software (version 3.4.1, R Foundation for Statistical Computing, 2017).

\section{Conclusions}

This study demonstrated the differentiated prognostic impact of D816V KIT mutation among various KIT mutations and clarified optimal time points and thresholds for RUNX1-RUNX1T1 MRD monitoring in the setting of HSCT. Both D816V KIT mutation and peri-transplant MRD monitoring with RUNX1-RUNX1T1 were independently associated with post-transplant relapse and survival. This study also revealed the relevance of each transplant type in each risk group by favorable outcomes of Auto-HSCT in MRD-negative patients without D816V KIT mutation and Allo-HSCT in patients with MRD positivity or D816V KIT mutation. Effects of transplant type in MRD-positive patients need to be further evaluated in a larger cohort. However, very poor outcomes in pre-transplant MRD-positive patients with D816V KIT suggested that this group should be treated in clinical trial and research. Finally, our data suggested that risk stratification by both D816V KIT mutation and RUNX1-RUNX1T1 MRD status could provide a platform for decision-making or risk-adapted therapeutic approaches.

Supplementary Materials: The following are available online at https:/ /www.mdpi.com/2072-669 4/13/2/336/s1. Figure S1: Trial profile; Figure S2: Cumulative incidence of relapse (CIR) according to types of KIT mutations; Figure S3: Survival outcomes according to D816V KIT mutation; Figure S4: Cumulative incidence of relapse according to various cutoffs of RUNX1-RUNX1T1 transcript levels at pre-HSCT and at 1 month or 3 months after HSCT: Figure S5: Survival outcomes according to MRD positivity defined by $3 \log$ reduction in RUNX1-RUNX1T1 levels at each time point; Figure S6: Survival outcomes according to transplant type; Figure S7: Survival outcomes in each group stratified by both D816V KIT mutations and the MRD positivity defined by 3 log reduction in RUNX1RUNX1T1 levels at pre-HSCT; Table S1: Patient-, disease-, and transplant-related characteristics 
according to transplant type; Table S2: Factors affecting survival outcomes (Univariate analysis); Table S3: Multivariate analysis to reveal an impact of KIT or D816 KIT mutations on survival outcomes; Table S4: Impact of transplant type on kinetics of RUNX1-RUNX1T1 transcript levels; Table S5: Sensitivity and specificity of RUNX1-RUNX1T1 MRD-positive patients defined by various cutoffs at each time point; Table S6: Multivariate analysis to reveal an impact of RUNX1-RUNX1T1 quantification at each time point on survival outcomes; Supplementary Methods.

Author Contributions: Conceptualization, B.-S.C. and H.-J.K.; data curation, G.-J.M., S.-S.P., S.P., K.-S.E., Y.-J.K., S.L., C.-K.M., S.-G.C., D.-W.K. and J.-W.L.; formal analysis, B.-S.C. and M.-S.K.; funding acquisition, B.-S.C. and H.-J.K.; investigation, G.-J.M., S.-H.S. and S.-A.Y.; methodology, B.-S.C., M.-S.K. and Y.-G.K.; resources, S.-S.P., Y.-W.J., J.-H.Y. and S.-E.L.; supervision, H.-J.K.; validation, H.-J.K.; visualization, G.-J.M. and S.P.; writing—original draft, B.-S.C., M.-S.K. and H.-J.K.; writing-review and editing, B.-S.C. and H.-J.K. All authors have read and agreed to the published version of the manuscript.

Funding: This research was supported by a grant from the Korea Health Technology R\&D Project through the Korea Health Industry Development Institute (KHIDI), funded by the Ministry of Health and Welfare, Republic of Korea (HI18C0480) and a grant from the National R\&D Program for Cancer Control, Ministry for Health and Welfare, Republic of Korea (HA17C0042).

Institutional Review Board Statement: The study was conducted according to the guidelines of the Declaration of Helsinki and approved by the Institutional Review Board of the Catholic Medical Center (protocol code: KC16TISI0438; approved 8 July 2016).

Informed Consent Statement: Patient consent was waived because formal consent is not required for this type of study.

Data Availability Statement: The data presented in this study are available on request from the corresponding author. The data are not publicly available due to ethical considerations.

Conflicts of Interest: The authors declare no competing financial interests.

\section{References}

1. Jourdan, E.; Boissel, N.; Chevret, S.; Delabesse, E.; Renneville, A.; Cornillet, P.; Blanchet, O.; Cayuela, J.M.; Recher, C.; Raffoux, E.; et al. Prospective evaluation of gene mutations and minimal residual disease in patients with core binding factor acute myeloid leukemia. Blood 2013, 121, 2213-2223. [CrossRef] [PubMed]

2. Marcucci, G.; Mrozek, K.; Ruppert, A.S.; Maharry, K.; Kolitz, J.E.; Moore, J.O.; Mayer, R.J.; Pettenati, M.J.; Powell, B.L.; Edwards, C.G.; et al. Prognostic factors and outcome of core binding factor acute myeloid leukemia patients with $\mathrm{t}(8 ; 21)$ differ from those of patients with inv(16): A Cancer and Leukemia Group B study. J. Clin. Oncol. 2005, 23, 5705-5717. [CrossRef] [PubMed]

3. Schlenk, R.F.; Benner, A.; Krauter, J.; Buchner, T.; Sauerland, C.; Ehninger, G.; Schaich, M.; Mohr, B.; Niederwieser, D.; Krahl, R.; et al. Individual patient data-based meta-analysis of patients aged 16 to 60 years with core binding factor acute myeloid leukemia: A survey of the German Acute Myeloid Leukemia Intergroup. J. Clin. Oncol. 2004, 22, 3741-3750. [CrossRef] [PubMed]

4. Cher, C.Y.; Leung, G.M.; Au, C.H.; Chan, T.L.; Ma, E.S.; Sim, J.P.; Gill, H.; Lie, A.K.; Liang, R.; Wong, K.F.; et al. Next-generation sequencing with a myeloid gene panel in core-binding factor AML showed KIT activation loop and TET2 mutations predictive of outcome. Blood Cancer J. 2016, 6, e442. [CrossRef] [PubMed]

5. Duployez, N.; Marceau-Renaut, A.; Boissel, N.; Petit, A.; Bucci, M.; Geffroy, S.; Lapillonne, H.; Renneville, A.; Ragu, C.; Figeac, M.; et al. Comprehensive mutational profiling of core binding factor acute myeloid leukemia. Blood 2016, 127, 2451-2459. [CrossRef]

6. Faber, Z.J.; Chen, X.; Gedman, A.L.; Boggs, K.; Cheng, J.; Ma, J.; Radtke, I.; Chao, J.R.; Walsh, M.P.; Song, G.; et al. The genomic landscape of core-binding factor acute myeloid leukemias. Nat. Genet. 2016, 48, 1551-1556. [CrossRef]

7. Kawashima, N.; Akashi, A.; Nagata, Y.; Kihara, R.; Ishikawa, Y.; Asou, N.; Ohtake, S.; Miyawaki, S.; Sakura, T.; Ozawa, Y.; et al. Clinical significance of ASXL2 and ZBTB7A mutations and C-terminally truncated RUNX1-RUNX1T1 expression in AML patients with $\mathrm{t}(8 ; 21)$ enrolled in the JALSG AML201 study. Ann. Hematol. 2019, 98, 83-91. [CrossRef]

8. Ishikawa, Y.; Kawashima, N.; Atsuta, Y.; Sugiura, I.; Sawa, M.; Dobashi, N.; Yokoyama, H.; Doki, N.; Tomita, A.; Kiguchi, T.; et al. Prospective evaluation of prognostic impact of KIT mutations on acute myeloid leukemia with RUNX1-RUNX1T1 and CBFBMYH11. Blood Adv. 2020, 4, 66-75. [CrossRef]

9. Kim, H.J.; Ahn, H.K.; Jung, C.W.; Moon, J.H.; Park, C.H.; Lee, K.O.; Kim, S.H.; Kim, Y.K.; Kim, H.J.; Sohn, S.K.; et al. KIT D816 mutation associates with adverse outcomes in core binding factor acute myeloid leukemia, especially in the subgroup with RUNX1/RUNX1T1 rearrangement. Ann. Hematol. 2013, 92, 163-171. [CrossRef] 
10. Rucker, F.G.; Agrawal, M.; Corbacioglu, A.; Weber, D.; Kapp-Schwoerer, S.; Gaidzik, V.I.; Jahn, N.; Schroeder, T.; Wattad, M.; Lubbert, M.; et al. Measurable residual disease monitoring in acute myeloid leukemia with t(8;21)(q22;q22.1): Results from the AML Study Group. Blood 2019, 134, 1608-1618. [CrossRef]

11. Krauth, M.T.; Eder, C.; Alpermann, T.; Bacher, U.; Nadarajah, N.; Kern, W.; Haferlach, C.; Haferlach, T.; Schnittger, S. High number of additional genetic lesions in acute myeloid leukemia with $t(8 ; 21) / R U N X 1-R U N X 1 T 1$ : Frequency and impact on clinical outcome. Leukemia 2014, 28, 1449-1458. [CrossRef] [PubMed]

12. Chen, W.; Xie, H.; Wang, H.; Chen, L.; Sun, Y.; Chen, Z.; Li, Q. Prognostic Significance of KIT Mutations in Core-Binding Factor Acute Myeloid Leukemia: A Systematic Review and Meta-Analysis. PLoS ONE 2016, 11, e0146614. [CrossRef] [PubMed]

13. Dohner, H.; Estey, E.; Grimwade, D.; Amadori, S.; Appelbaum, F.R.; Buchner, T.; Dombret, H.; Ebert, B.L.; Fenaux, P.; Larson, R.A.; et al. Diagnosis and management of AML in adults: 2017 ELN recommendations from an international expert panel. Blood 2017, 129, 424-447. [CrossRef] [PubMed]

14. Yu, G.; Yin, C.; Wu, F.; Jiang, L.; Zheng, Z.; Xu, D.; Zhou, J.; Jiang, X.; Liu, Q.; Meng, F. Gene mutation profile and risk stratification in AML1ETOpositive acute myeloid leukemia based on nextgeneration sequencing. Oncol. Rep. 2019, 42, 2333-2344. [CrossRef] [PubMed]

15. Schuurhuis, G.J.; Heuser, M.; Freeman, S.; Bene, M.C.; Buccisano, F.; Cloos, J.; Grimwade, D.; Haferlach, T.; Hills, R.K.; Hourigan, C.S.; et al. Minimal/measurable residual disease in AML: A consensus document from the European LeukemiaNet MRD Working Party. Blood 2018, 131, 1275-1291. [CrossRef]

16. Wang, Y.; Wu, D.P.; Liu, Q.F.; Qin, Y.Z.; Wang, J.B.; Xu, L.P.; Liu, Y.R.; Zhu, H.H.; Chen, J.; Dai, M.; et al. In adults with t(8;21)AML, posttransplant RUNX1/RUNX1T1-based MRD monitoring, rather than c-KIT mutations, allows further risk stratification. Blood 2014, 124, 1880-1886. [CrossRef]

17. Yin, J.A.; O’Brien, M.A.; Hills, R.K.; Daly, S.B.; Wheatley, K.; Burnett, A.K. Minimal residual disease monitoring by quantitative RT-PCR in core binding factor AML allows risk stratification and predicts relapse: Results of the United Kingdom MRC AML-15 trial. Blood 2012, 120, 2826-2835. [CrossRef]

18. Zhu, H.H.; Zhang, X.H.; Qin, Y.Z.; Liu, D.H.; Jiang, H.; Chen, H.; Jiang, Q.; Xu, L.P.; Lu, J.; Han, W.; et al. MRD-directed risk stratification treatment may improve outcomes of $t(8 ; 21)$ AML in the first complete remission: Results from the AML05 multicenter trial. Blood 2013, 121, 4056-4062. [CrossRef]

19. Gorin, N.C.; Labopin, M.; Frassoni, F.; Milpied, N.; Attal, M.; Blaise, D.; Meloni, G.; Iori, A.P.; Michallet, M.; Willemze, R.; et al. Identical outcome after autologous or allogeneic genoidentical hematopoietic stem-cell transplantation in first remission of acute myelocytic leukemia carrying inversion 16 or $\mathrm{t}(8 ; 21)$ : A retrospective study from the European Cooperative Group for Blood and Marrow Transplantation. J. Clin. Oncol. 2008, 26, 3183-3188. [CrossRef]

20. Nakasone, H.; Izutsu, K.; Wakita, S.; Yamaguchi, H.; Muramatsu-Kida, M.; Usuki, K. Autologous stem cell transplantation with PCR-negative graft would be associated with a favorable outcome in core-binding factor acute myeloid leukemia. Biol. Blood Marrow Transplant. 2008, 14, 1262-1269. [CrossRef]

21. Kuwatsuka, Y.; Miyamura, K.; Suzuki, R.; Kasai, M.; Maruta, A.; Ogawa, H.; Tanosaki, R.; Takahashi, S.; Koda, K.; Yago, K.; et al. Hematopoietic stem cell transplantation for core binding factor acute myeloid leukemia: $\mathrm{T}(8 ; 21)$ and inv(16) represent different clinical outcomes. Blood 2009, 113, 2096-2103. [CrossRef] [PubMed]

22. Eom, K.S.; Kim, H.J.; Cho, B.S.; Lee, S.E.; Yahng, S.A.; Yoon, J.H.; Shin, S.H.; Jeon, Y.W.; Kim, J.H.; Kim, Y.J.; et al. Equivalent outcome of autologous stem cell transplantation and reduced intensity conditioning stem cell transplantation in acute myeloid leukemia patients with t(8;21). Acta Haematol. 2015, 133, 266-276. [CrossRef] [PubMed]

23. Shin, H.J.; Min, W.S.; Min, Y.H.; Cheong, J.W.; Lee, J.H.; Kim, I.H.; Hong, D.S.; Ahn, J.S.; Kim, H.J.; Lee, W.S.; et al. Different prognostic effects of core-binding factor positive AML with Korean AML registry data. Ann. Hematol. 2019, 98, 1135-1147. [CrossRef] [PubMed]

24. Al-Harbi, S.; Aljurf, M.; Mohty, M.; Almohareb, F.; Ahmed, S.O.A. An update on the molecular pathogenesis and potential therapeutic targeting of AML with t(8;21)(q22;q22.1);RUNX1-RUNX1T1. Blood Adv. 2020, 4, 229-238. [CrossRef] [PubMed]

25. Hansrivijit, P.; Gale, R.P.; Barrett, J.; Ciurea, S.O. Cellular therapy for acute myeloid Leukemia-Current status and future prospects. Blood Rev. 2019, 37, 100578. [CrossRef]

26. Greiner, J.; Gotz, M.; Bunjes, D.; Hofmann, S.; Wais, V. Immunological and Clinical Impact of Manipulated and Unmanipulated DLI after Allogeneic Stem Cell Transplantation of AML Patients. J. Clin. Med. 2019, 9, 39. [CrossRef]

27. Tarlock, K.; Alonzo, T.A.; Wang, Y.C.; Gerbing, R.B.; Ries, R.; Loken, M.R.; Pardo, L.; Hylkema, T.; Joaquin, J.; Sarukkai, L.; et al. Functional Properties of KIT Mutations Are Associated with Differential Clinical Outcomes and Response to Targeted Therapeutics in CBF Acute Myeloid Leukemia. Clin. Cancer Res. 2019, 25, 5038-5048. [CrossRef]

28. Willekens, C.; Blanchet, O.; Renneville, A.; Cornillet-Lefebvre, P.; Pautas, C.; Guieze, R.; Ifrah, N.; Dombret, H.; Jourdan, E.; Preudhomme, C.; et al. Prospective long-term minimal residual disease monitoring using RQ-PCR in RUNX1-RUNX1T1-positive acute myeloid leukemia: Results of the French CBF-2006 trial. Haematologica 2016, 101, 328-335. [CrossRef]

29. Opatz, S.; Bamopoulos, S.A.; Metzeler, K.H.; Herold, T.; Ksienzyk, B.; Braundl, K.; Tschuri, S.; Vosberg, S.; Konstandin, N.P.; Wang, C.; et al. The clinical mutatome of core binding factor leukemia. Leukemia 2020, 34, 1553-1562. [CrossRef]

30. Derman, B.A.; Larson, R.A. Post-remission therapy in acute myeloid leukemia: Are we ready for an individualized approach? Best Pract. Res. Clin. Haematol. 2019, 32, 101102. [CrossRef] 
31. Appelbaum, F.R.; Kopecky, K.J.; Tallman, M.S.; Slovak, M.L.; Gundacker, H.M.; Kim, H.T.; Dewald, G.W.; Kantarjian, H.M.; Pierce, S.R.; Estey, E.H. The clinical spectrum of adult acute myeloid leukaemia associated with core binding factor translocations. Br. J. Haematol. 2006, 135, 165-173. [CrossRef] [PubMed]

32. Byrd, J.C.; Weiss, R.B.; Arthur, D.C.; Lawrence, D.; Baer, M.R.; Davey, F.; Trikha, E.S.; Carroll, A.J.; Tantravahi, R.; Qumsiyeh, M.; et al. Extramedullary leukemia adversely affects hematologic complete remission rate and overall survival in patients with t(8;21)(q22;q22): Results from Cancer and Leukemia Group B 8461. J. Clin. Oncol. 1997, 15, 466-475. [CrossRef] [PubMed]

33. Yoon, J.H.; Kim, H.J.; Kim, J.W.; Jeon, Y.W.; Shin, S.H.; Lee, S.E.; Cho, B.S.; Eom, K.S.; Kim, Y.J.; Lee, S.; et al. Identification of molecular and cytogenetic risk factors for unfavorable core-binding factor-positive adult AML with post-remission treatment outcome analysis including transplantation. Bone Marrow Transplant. 2014, 49, 1466-1474. [CrossRef]

34. Yoon, J.H.; Kim, H.J.; Park, S.S.; Jeon, Y.W.; Lee, S.E.; Cho, B.S.; Eom, K.S.; Kim, Y.J.; Lee, S.; Min, C.K.; et al. Long-term clinical outcomes of hematopoietic cell transplantation for intermediate-to-poor-risk acute myeloid leukemia during first remission according to available donor types. Oncotarget 2017, 8, 41590-41604. [CrossRef] [PubMed]

35. Gyurkocza, B.; Sandmaier, B.M. Conditioning regimens for hematopoietic cell transplantation: One size does not fit all. Blood 2014, 124, 344-353. [CrossRef]

36. Kim, Y.; Lee, G.D.; Park, J.; Yoon, J.H.; Kim, H.J.; Min, W.S.; Kim, M. Quantitative fragment analysis of FLT3-ITD efficiently identifying poor prognostic group with high mutant allele burden or long ITD length. Blood Cancer J. 2015, 5, e336. [CrossRef] 